\title{
Adaptive Routing Tree Construction for Achieving Optimal Throughput in WiMAX Mesh Networks
}

\author{
Hyungwoo Choi*, Tae-hwa Kim*, Hong-Shik Park* \\ *Department of Information \& Communications Engineering, KAIST, Daejeon, Republic of Korea \\ neojoey@kaist.ac.kr, thkim0@kaist.ac.kr, parkhs@kaist.ac.kr
}

\begin{abstract}
This paper explores the trade-off relationship between a spatial diversity gain and a control overhead with regard to the number of hop count of the routing tree in WiMAX mesh networks. The effective gain or overhead with the number of hop count is influenced by the density of SSs and traffic load. So this paper proposes the algorithm adjusting the depth of routing tree according to the time varying number of SSs and traffic load for achieving optimal throughput performance. The simulation results show the proposed algorithm can have throughput performance enhancement in the time varying traffic load environment.
\end{abstract}

Keywords - WiMAX, mesh mode, routing tree construction.

\section{INTRODUCTION}

The IEEE 802.16 also known as WiMAX (Worldwide Interoperability for Microwave Access) defines two kind of operation mode: Point to Multi-point (PMP) mode and Mesh mode [1]. In PMP mode, the Subscriber Stations (SSs) communicate only with the serving Base Station (BS). In contrast to the PMP mode, in Mesh mode, SSs can communicate with not only BS but SSs themselves and BS acts as a gateway between the mesh network and the outer network. The mesh mode can provide larger cell capacity, more increased coverage, and higher robustness to non-lineof-sight transmission impairment than the PMP mode.

The IEEE 802.16 mesh mode operates with two kinds of resource allocation strategies: distributed scheduling and centralized scheduling. In the centralized scheduling, a tree topology rooted by the BS which acts as a cluster head must be established to describe the routing information between the BS and SSs. The BS has the responsibility for the bandwidth allocation and the time slot assignment for each SS. In addition, it periodically collects the network configuration and bandwidth requirement of each SS and arranges a schedule to avoid collision, reduce the transmission cycle, and maximize network throughput. In the distributed scheduling, it works based on the Election Based Transmission Timing (EBTT) procedure which is used to manage the control slots allocation. The connections setup up with neighbors by the 3-way handshaking. Since the centralized scheduling maintains the global information such as the network topology and the bandwidth requirements of all SSs, it is more efficient and easier to implement than distributed scheduling [2].
The one of the major issues addressed in many studies is the capacity enhancement in wireless multi-hop networks. Achieving spatial reuse with concurrency among multi-hop transmissions is the one of the solutions for the capacity enhancement in such environment. However, the interference is a major problem in concurrent transmission for the multihop mesh networks. Another major issue is the structure of the routing tree which plays important role and enhances the performance of centralized scheduling. According to the structure of routing tree, it could reduce the interference between links and balance the traffic load.

In [3], an interference-aware routing tree construction algorithm and an enhanced centralized mesh scheduling scheme achieving high spectral utilization are introduced. The proposed scheduling scheme considers selecting the routing paths with less interference so as to improve the throughput. To achieve this, the authors define a blocking metric for a route which is used during the route construction, modelling the number of nodes whose transmission will be blocked by the route. For this, interference level of routes in the mesh is modelled. Each joining mode selects its sponsor node among its neighbors with the minimum interference level.

[4]-[6] extend the idea based on [3], [4] redefines the blocking metric which is defined to be the number of blocked nodes multiplied by the number of packets at a node. In [5], they consider the delay of relaying data and determine the order of transmission time, in which nodes with the biggest hop-count comes first, ones with the same hop-count keep their orders. Also, [6] focuses on the tree structure to handle centralized scheduling to achieve reduction in the length of scheduling, improvement in the channel utilization ratio and decrease the transmission delay. In allocation of slots, link selection can be done in four ways - random, min-interference, nearest to BS and farthest to BS, rather than allocating for the link with the highest unallocated traffic demand as in [3].

In [7], the authors analyse the design trade-offs between the depth and fan-out of the mesh tree construction. Long links increase the distance that can be served while being able to support only low bit rate whereas shorter links work with higher rates, since IEEE 802.16 standard adapts Adaptive Modulation and Coding (AMC) scheme. Reducing the depth of tree reduces the number of transmissions needed for the same packet hence reduces the control packet overhead. Also, if the distance is longer, the needed transmission power is 
higher which in turn causes higher interference and decreases the chance of having spatial reuses. In contrast, increasing the depth increases the data rate but reduces the distance. As the tree depth increases, the control overhead reduces the available slot space for data because low bit-rate but robust modulation and coding level is used for the control header, thus decreasing the achievable throughput. They recommend having deeper trees which split long links into multiple smaller links considering the simulation results obtained.

Since the depth of routing tree can influence the throughput of the wireless mesh networks as shown in [7]. The tree structure should be carefully managed in order to have an optimal performance. The deeper tree does not always show better performance in certain circumstance. The traffic load and density of SSs can fluctuate significantly in spatial and temporal situation. For example, the traffic load in the daytime is relatively heavy in office areas and light in residential areas, while the opposite things happen in the evening. So, if the number of SSs (or the density of SSs) in the network is low, it has low chance to take advantage of the spatial diversity gain and low bit-rate according to the average hop-distance being farther. Also, deep-depth routing tree makes the control overhead relatively heavier. On the other hand, if many SSs are in the network, the value of the spatial diversity gain over the control overhead is much higher compared to the former case. So, the deep-depth routing tree is more effective in this case.

In this paper, we consider the both of spatial diversity gain and control overhead as parameters of the algorithm and propose the algorithm adjusting the depth of routing tree according to the time varying number of SSs and traffic load. In section II, we describe assumption and propose our algorithm. In section III, we analyse the simulation results and performance. Lastly, the conclusions are drawn in section IV.

\section{Adaptive Routing Tree Construction Mechanism}

We try to adjust the depth of the routing tree (the maximum number of hop counts) of the mesh network by changing the candidates of first-hop nodes which are nearby the BS. The nodes within the BS utilize their own AMC level by the received power which depends on the distance between BS and themselves. So, we adjust the candidates of first-hop nodes of the routing tree according to the density of SSs in the network. The example situation is shown in the Figure 1.

\section{A. System model}

We suppose Orthogonal Frequency Division Multiplexing (OFDM) in the physical layer and utilize the original messages defined for mesh mode in IEEE 802.16 standard such as Mesh Network Configuration (MSH-NCFG) message, Mesh Network Entry (MSH-NENT) message, Mesh Centralized Scheduling (MSH-CSCH) message and Mesh Centralized Scheduling Configuration (MSH-CSCF) message.[1] We assume the nomadic mobility of SSs which means SSs does not frequently move and has relatively long residual time once they moved. So, we assume that the

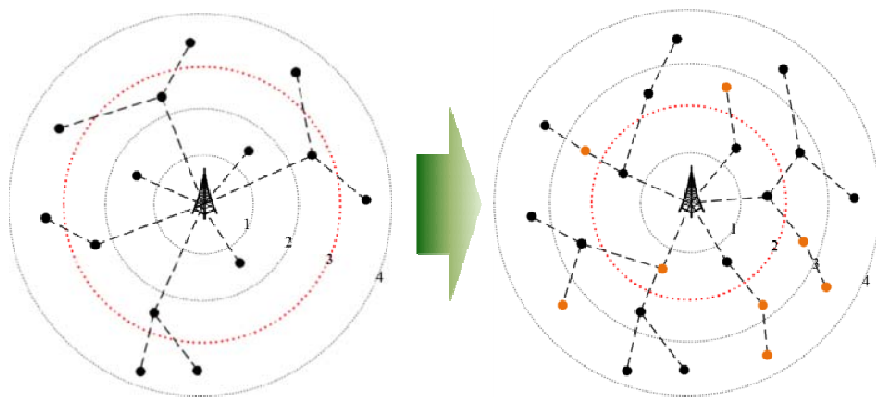

Figure 1. An example routing tree construction when the density of SSs has been chnaged (the color dots are new coming SSs)

network situation changes in the long term period such as day time/evening time for a day.

For a convenience, we consider a single BS in the network and downlink traffic which is received from the Internet. There is the number of $N$ SSs which are uniformly distributed in the network. Each of SSs has a fixed communication range of $\mathrm{R}$ and receives traffic with the same rate of $\lambda$ from the Internet. We will use the SS and the node interchangeably in this paper. The size of the control sub-frame is $7 \times \mathrm{MSH}$ CTRL-LEN OFDM symbols. The maximum depth of mesh tree is 7 since MSH-NCFG has 3-bit field for the number of hops. Also, the AMC level has 7 steps: BPSK1/2, QPSK1/2, QPSK3/4, 16QAM 1/2, 16QAM 3/4, 64QAM 2/3, and 64QAM 3/4. $A_{i}$ denotes the set of SSs whose AMC level is $i$ and the cardinality of $A_{i}$ is the number of SSs with AMC level $i$

$$
A=\left[A_{1}, A_{2}, \ldots, A_{7}\right] .
$$

In the IEEE 802.16 mesh mode, the traffic flow recursively. Assuming the quasi-balanced tree [7] where the number of children of each SS is variable and the average number of children is $L$. The number of OFDM symbols in each data sub-frame transmission opportunity is determined by

$$
S_{\text {data }}=\left\lceil\frac{T_{f} / T_{S}-7 \times M S H_{-} C T R L_{-} L E N}{256}\right\rceil,
$$

where $T_{f}$ is the frame duration and $T_{S}$ is the OFDM symbol duration [8]. Then, the $S_{\text {data }}$ is allocated proportionally to the SSs at level $k$ as

$$
L^{k} / \sum_{k=1}^{n} L^{k}
$$

where the number of SSs in level $k$ of tree is $L^{k}$.

The average traffic of link $j$ at level $k$ of the tree is defined as

$$
c_{j, k}=\left(\sum_{i=1}^{k} L^{i} \times \lambda\right) / L^{k} .
$$

The number of OFDM symbols which is required to transmit the traffic $c_{j, k}$ of the link $j$ at level $k$ of the tree in the frame $d_{j, k}$ is determined by

$$
d_{j, k}=\left\lceil\frac{c_{j, k} T_{f} / b_{j}+h}{S_{\text {data }}}\right\rceil \times S_{\text {data }},
$$

where $h$ is the number of overhead slots, $b_{j}$ is the number of bits in each OFDM symbol. The link between the BS and the first-hop nodes of the routing tree plays the role of backhaul link of the children nodes in the mesh networks. When the first-hop nodes are within the AMC level $m$, the link capacity of the first-hop nodes in the tree is defined as 


$$
C_{f i r s t}=\frac{\sum_{i=1}^{m}\left(\left|A_{i}\right| \cdot \frac{b_{A_{i}}}{T_{f}}\right)}{\sum_{i=1}^{m}\left|A_{i}\right|} \cdot\left(d_{j, 1}-h\right) \cdot L
$$

Also, the total capacity of children nodes of the tree with maximum tree depth $n$ is defined as

$$
C_{\text {children }}=\sum_{k=2}^{n}\left(d_{j, k}-h\right) \frac{b_{j, k}}{T_{f}} \times \alpha,
$$

where $\alpha$ is the concurrent parameter we defined. To transmit the traffic concurrently, SSs should be located disjointly. Since each of SS has fixed communication range $R$ and normally the interference reaches range of $2 R$, the average number of disjoint area except the first-hop of the tree in the network is calculated as

$$
\alpha=\frac{\left(r_{\max }^{2}-r_{f}^{2}\right)}{(2 R)^{2}},
$$

where $r_{\max }$ is maximum radius of the mesh network, and $r_{f}$ is the radius of the first hop of the tree. So, the total capacity of the mesh network is

$$
C_{\text {Mesh }}=C_{\text {first }}+C_{\text {children }} .
$$

\section{B. Adaptive routing tree construction algorithm}

To achieve the optimal throughput of the mesh network, we have to decide the first-hop area in $A_{m}$ and the depth of the routing tree in depth $n$.

$$
\arg \max _{i} C_{\text {Mesh }}
$$

where $i \in\{1,2, \ldots, 7\}$, since the AMC level is divided in 7 steps. So, we can easily get the value of $A_{m}$ from (10).

The total throughput of the mesh network can be varied according to the density of SSs in the network. By utilizing the value of $A_{m}$ and depth $n$, we can adjust the routing tree which maximize the throughput of the network.

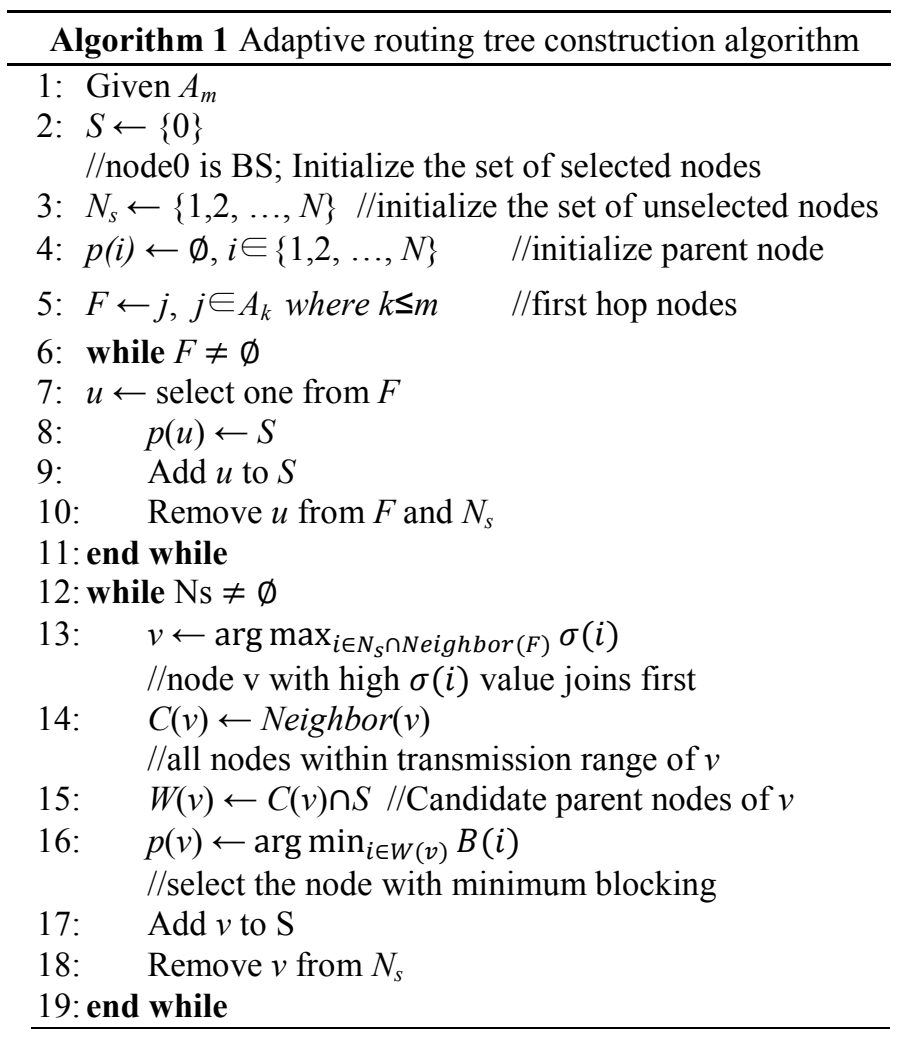

The time sequence of node $i$ joining the network is defined by $\sigma(i)$ and $B(i)$ is the blocking metric of a node $i$ like as defined in [3]. $B(i)$ is the number of blocked nodes by all the intermediate nodes along the route when node $i$ is transmit the traffic.

\section{SiMULATION RESULTS}

For the simulations, we deploy the SSs in the quasibalanced tree topology rooted by BS with the average number of children node $L$ and the maximum level of tree equals 7 . We set the frame duration $T_{f}=10 \mathrm{~ms}$ and the bandwidth of the OFDM to $20 \mathrm{MHz}$. So, the duration of OFDM symbols $T_{S}=$ $12.5 \mu \mathrm{s}$. The bits rate per OFDM symbol of each AMC level is set to [ 96, 192, 288, 384, 576, 768, 864 ] (bits/symbol) respectively and the length of control sub-frame MSH-CTRLLEN is set to 10 .

We simulated by changing the value of $L$. Since the SSs are located in quasi-balanced tree topology, we suppose that the density of SSs in the network varies with the value of $L$ and each of SSs receives same amount of data with the rate of $\lambda$. In the varying the density of SSs situation, we construct the routing tree. We simulated by changing the set of the first-hop nodes which communicate with BS directly. For example, at first, the nodes in the first level of the tree topology are being the first-hop nodes and then the nodes in the next second level of the tree topology are being the boundary of the next set of first-hop nodes, and so on.

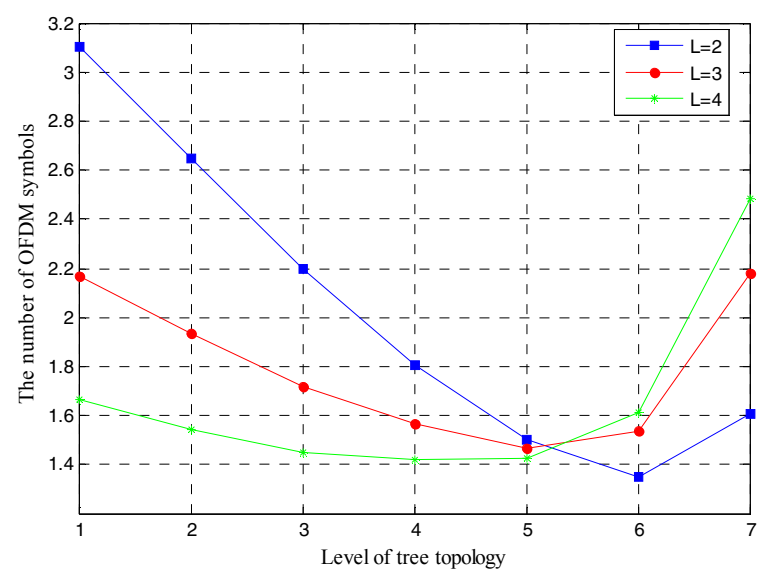

Figure 2. The number of normalized OFDM symbols to serve the given traffic in low density of SSs

The results of the simulation are shown in Figure 2 and Figure 3. They show the normalized number of OFDM symbols used to serve the traffic which is given to the mesh network in low and high density of SSs respectively. As the density of SSs in the networks grows, the set of first-hop nodes is being closer to BS. This means that low-depth of the routing tree is more efficient for the low density of SSs, while the deep-depth of the routing tree is more efficient for the high density of SSs in the networks. For example, in case of $L=2$, which means there $\sum_{k=1}^{7} 2^{k}$ SSs are in the networks, it consumes smallest amount of OFDM symbols when the nodes within the level 6 of tree topology become the first-hop node 
of the routing tree. In other words, it can achieve the optimal throughput by making the nodes within the level 6 of tree topology the first-hop nodes of the routing tree.

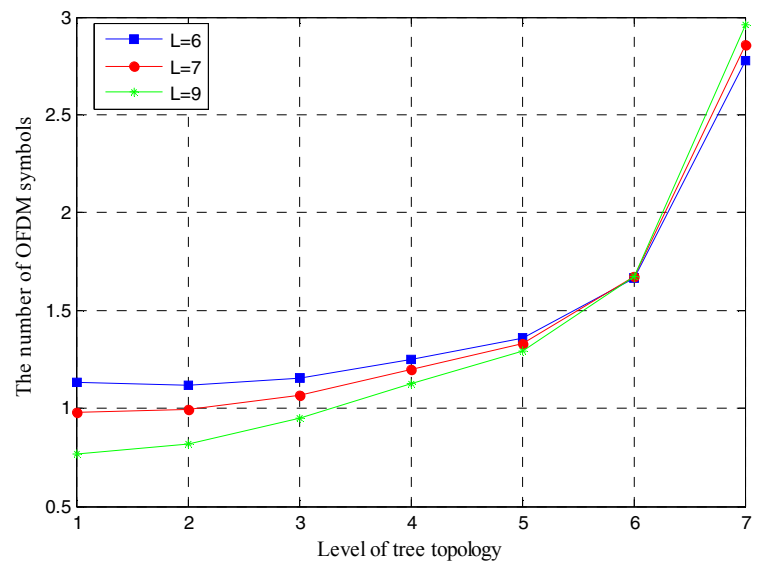

Figure 3. The number of normalized OFDM symbols to serve the given traffic in high density of SSs

When the density of SSs is relatively higher such as $L=6$ or 9 shown in Figure 3, the optimal throughput can be achieved as the set of first-hop nodes is closer to the BS.

\section{IV.CONCLUSIONS}

In this paper, we explore the trade-off relationship between a spatial diversity gain and a control overhead with regard to the number of hop count of the routing tree in WiMAX mesh networks. Also, we propose the algorithm which adjusts the depth of routing tree of mesh networks according to the time varying number of SSs and traffic load. The simulation results shows that adjusting the depth of routing tree according to the network situations can achieve the optimal throughput performance. By the simulation, we can say that the low-depth of the routing tree is more efficient for the low density of SSs, while the deep-depth of the routing tree is more efficient for the high density of SSs in the networks. For the further works, we will analyse the QoS parameters such as delay or jitter with our algorithm in the same situation.

\section{ACKNOWLEDGMENT}

This research was funded by the MSIP (Ministry of Science, ICT \& Future Planning), Korea in the ICT R\&D Program 2013.

\section{REFERENCES}

[1] IEEE Standard for Local and Metropolitan Area Networks Part 16: Air Interface for Fixed Broadband Wireless Access Systems, IEEE Std 802.16-2004 (Revision of IEEE Std 802.16-2001), vol., no., pp.0_1857, 2004.

[2] M. Kas, B. Yargicoglu, I. Korpeoglu, and E. Karasan, "A Survey on Scheduling in IEEE 802.16 Mesh Mode," Communications Surveys \& Tutorials, IEEE, vol.12, no.2, pp.205-221, Second Quarter 2010.

[3] H. Wei, S. Ganguly, R. Izmailov, and Z.J. Haas, "Interference-aware IEEE 802.16 WiMax mesh networks," Vehicular Technology Conference, 2005. VTC 2005-Spring. 2005 IEEE 61st, vol.5, no., pp.3102-3106 Vol. 5, 30 May-1 June 2005.

[4] J. Tao, F. Liu, Z. Zeng, and Z. Lin, "Throughput enhancement in WiMax mesh networks using concurrent transmission," Wireless Communications, Networking and Mobile Computing, 2005. Proceedings. 2005 International Conference on, vol.2, no., pp.871-874, 23-26 Sept. 2005.

[5] F. Jin, A. Arora, J. Hwang, and H.-A. Choi, "Routing and packet scheduling in WiMAX mesh networks," Broadband Communications, Networks and Systems, 2007. BROADNETS 2007. Fourth International Conference on, vol., no., pp.574-582, 10-14 Sept. 2007.

[6] B. Han, F. Tso, L. Lin, and W. Jia, "Performance Evaluation of Scheduling in IEEE 802.16 Based Wireless Mesh Networks," Mobile Adhoc and Sensor Systems (MASS), 2006 IEEE International Conference on , vol., no., pp.789-794, Oct. 2006.

[7] S. Nahle, L. Iannone, B. Donnet, and T. Friedman, "Investigating depth-fanout trade-off in WiMAX mesh networks." 1st WEIRD Workshop on WiMAX, Wireless and Mobility. 2007.

[8] P. Djukic, and S. Valaee. "Centralized scheduling algorithms for 802.16 mesh networks." Wimax/MobileFi: Advanced research and technology (2007). 